\title{
On a free boundary problem modelling inductive-heating processes
}

\author{
HONG-MING YIN ${ }^{\dagger}$ \\ Department of Mathematics, Washington State University, Pullman, WA 99164, USA
}

[Received 26 February 2007 and in revised form 6 December 2007]

\begin{abstract}
We study a free boundary problem describing a melting process by using induction heating. The mathematical model in one space dimension consists of a coupled parabolic system in each phase along with a nonequilibrium kinetic condition on the interface. By applying an energy estimate and Campanato type estimates, it is shown that the problem has a unique classical solution globally.
\end{abstract}

2000 Mathematics Subject Classification: 35R35.

Keywords: Free boundary problem; induction heating; superheating.

\section{Introduction}

Superheating is a phenomenon in which the temperature in a solid phase is higher than the melting temperature. This phenomenon is common when a microwave or an induction heating method is used during a melting process. The classical interface conditions (Stefan conditions) have to be modified in order to model this phenomenon. In this paper we study a melting process for highly conductive materials by using an induction heating method with possible superheating in the process.

To derive the mathematical model, we recall Maxwell's equations for an electric field $\mathbf{E}(x, t)$ and a magnetic field $\mathbf{H}(x, t)$ in $\mathbb{R}^{3}$ ([11, 16]):

$$
\varepsilon \mathbf{E}_{t}+\sigma \mathbf{E}=\nabla \times \mathbf{H}, \quad \mu \mathbf{H}_{t}+\nabla \times \mathbf{E}=0,
$$

where $\varepsilon$ is the electric permittivity, $\mu$ the magnetic permeability and $\sigma$ the electric conductivity, which may depend on temperature. Here a bold letter represents a vector or vector function in $\mathbb{R}^{3}$.

Since the targeted material is highly conductive, the eddy currents, $\mathbf{J}(x, t):=\sigma \mathbf{E}(x, t)$, are much stronger than the displacement currents, $\varepsilon \mathbf{E}_{t}$. It is a common approximation for induction heating modelling ([16]) that one can neglect the displacement currents by simply setting $\varepsilon=0$. Hence, Maxwell's equations become a single system for $\mathbf{H}(x, t)$ :

$$
\mu \mathbf{H}_{t}+\nabla \times[\rho \nabla \times \mathbf{H}]=0
$$

where $\rho:=1 / \sigma$ represents the resistivity of the material which may depend on temperature as well as on space and time variables.

The local density of Joule's heat produced by eddy currents equals ([15, 16])

$$
\mathbf{E} \cdot \mathbf{J}=\rho|\nabla \times \mathbf{H}|^{2} .
$$

†E-mail: hyin@math.wsu.edu 
For simplicity, the heat convection is neglected here. By Fourier's law and the conservation of energy, we see that the temperature, denoted by $u$, satisfies

$$
u_{t}-\nabla[k \nabla u]=\rho|\nabla \times \mathbf{H}|^{2},
$$

where $k$ represents the thermal conductivity and the other physical constants such as the material density and specific heat are assumed to be constants.

The above coupled system for $\mathbf{H}$ and $u$ has been studied by many researchers in the engineering sciences (see [15, 16] and the references therein). Despite the importance of the model in applications, many mathematical issues are still open due to the complicated nonlinear coupling and degeneracy of the system for $\mathbf{H}(x, t)$. Some progress has been made during the past few years. The author [24] proved the global existence of a weak solution in $\mathbb{R}^{3}$ under the assumption that $\rho(u)$ is bounded with a positive lower bound (see also [3] for a similar result). The regularity of weak solutions is studied in [25]. In [24] (see also [10]), by deriving a De Giorgi-Nash type estimate for the steady-state system of the magnetic field, classical solvability is established for the coupled steady-state system for $u(x)$ and $\mathbf{H}(x)$.

The problem, however, becomes much more difficult when a phase-change occurs in the system. Some industrial problems can be reduced to or approximated by the one-dimensional case ([16]). For example, assume that an electric field is restricted along the $z$-direction and its value depends only on $x$, i.e., $\mathbf{E}(x, t)=\{0,0, e(x, t)\}$. Then the magnetic field must be in the $y$-direction, $\mathbf{H}(x, t)=\{0, h(x, t), 0\}$. The system for $\mathbf{H}$ becomes the following equation:

$$
h_{t}-\frac{\partial}{\partial x}\left[\rho(x, t, u) \frac{\partial h}{\partial x}\right]=0 .
$$

Since the spatial dimension is equal to one, we may assume that the interface between the liquid and solid materials can be expressed by $x=s(t), t \geqslant 0$. The classical Stefan condition at the interface is given by

$$
u(s(t)-, t)=u(s(t)+, t)=m, \quad L s^{\prime}(t)=-k_{1} u_{x}(s(t)-, t)+k_{2} u_{x}(s(t)+, t),
$$

where $m$ is the melting temperature, $L$ is the latent heat, and the constants $k_{1}$ and $k_{2}$ represent thermal conductivities in the solid and liquid, respectively. Here we denote by $f(s-)$ and $f(s+)$ the left- and right-hand limit of a function $f(x)$ as $x \rightarrow s$.

Because of the internal heat source produced by electromagnetic waves, the superheating phenomenon may occur. This leads to a nonequilibrium state at the interface, and the interface is unstable and unrealistic. Moreover, a mushy region may occur. In this paper, however, we only consider the classical case and assume that no mushy region occurs (see [29] for a different model). In order to stabilize the interface, several different theories have been proposed (see [4, 21] for examples). In this paper we use the following kinetic condition proposed in [21] from the nonequilibrium thermodynamics:

$$
\begin{aligned}
& u(s(t)-, t)=u(s(t)+, t), \\
& L s^{\prime}(t)=-k_{1} u_{x}(s(t)-, t)+k_{2} u_{x}(s(t)+, t), \\
& s^{\prime}(t)=-g(u(s(t), t)),
\end{aligned}
$$

where $g(u)$ is a known nonnegative function of $u$, which represents the nonequilibrium force on the interface. The sign condition on $g$ is to ensure that the model describes a melting process. 
Let $T>0$ and

$$
Q_{T}=\{(x, t): 0<x<1,0<t \leqslant T\}
$$

The interface is denoted by

$$
\Gamma_{T}=\{(x, t): x=s(t), 0 \leqslant t \leqslant T\} .
$$

The solid and liquid phases are denoted by

$$
Q_{T}^{-}=\{(x, t): 0<x<s(t)\}, \quad Q_{T}^{+}=\{(x, t): s(t)<x<1,0<t<T\} .
$$

Now we assume that the electric fields in the $z$-axis direction, denoted by $f_{1}(t)$ and $f_{2}(t)$, are applied on the fixed boundaries $x=0$ and $x=1$, respectively. From the relation between the electric and magnetic fields (Maxwell's first equation with $\varepsilon=0$ ), we see that

$$
\rho h_{x}(0, t)=f_{1}(t), \quad \rho h_{x}(1, t)=f_{2}(t) .
$$

For the temperature $u(x, t)$, we assume that there is no heat exchange across the fixed boundary.

We summarize the above discussion to obtain the following mathematical model for a melting process by using induction heating: Find $(h(x, t), u(x, t))$ and $s(t)$ such that

$$
\begin{aligned}
& h_{t}-\frac{\partial}{\partial x}\left[\rho(x, t, u) \frac{\partial h}{\partial x}\right]=0, \quad(x, t) \in Q_{T} \backslash \Gamma_{T}, \\
& u_{t}-\frac{\partial}{\partial x}\left[k(x, t, u) \frac{\partial u}{\partial x}\right]=\rho(x, t, u) h_{x}^{2}, \quad(x, t) \in Q_{T} \backslash \Gamma_{T}, \\
& h(s(t)-, t)=h(s(t)+, t), u(s(t)-, t)=u(s(t)+, t), \quad 0<t<T, \\
& \rho_{1} h_{x}(s(t)-, t)=\rho_{2} h_{x}(s(t)+, t), \quad 0<t<T, \\
& L s^{\prime}(t)=-k_{1} u_{x}(s(t)-, t)+k_{2} u_{x}(s(t)+, t), \quad s(0)=s_{0}, \quad 0<t<T, \\
& s^{\prime}(t)=-g(u(s(t), t)), \quad 0<t<T, \\
& \rho_{1}(0, t, u(0, t)) h_{x}(0, t)=f_{1}(t), \quad 0<t<T, \\
& \rho_{2}(1, t, u(1, t)) h_{x}(1, t)=f_{2}(t), \quad 0<t<T, \\
& u_{x}(0, t)=u_{x}(1, t)=0, \quad 0<t<T, \\
& h(x, 0)=h_{0}(x), \quad u(x, 0)=u_{0}(x), \quad 0<x<1,
\end{aligned}
$$

where $s_{0} \in(0,1) ; f_{1}(t), f_{2}(t)$ are prescribed electric fields on the fixed boundary; $u_{0}(x)$ and $h_{0}(x)$ represent the initial temperature and initial magnetic field; and

$$
k= \begin{cases}k_{1}(x, t, u) & \text { if }(x, t) \in Q_{T}^{-}, \\ k_{2}(x, t, u) & \text { if }(x, t) \in Q_{T}^{+},\end{cases}
$$

and

$$
\rho(x, t, u)= \begin{cases}\rho_{1}(x, t, u) & \text { if }(x, t) \in Q_{T}^{-}, \\ \rho_{2}(x, t, u) & \text { if }(x, t) \in Q_{T}^{+},\end{cases}
$$

During the past several decades, various free boundary problems have been studied by many authors (see the monographs and conference proceedings [14, 18, 17] for examples). Several authors 
have studied the superheating and supercooling phenomena (see [7, 8, 22] etc.). For the Stefan problem with a kinetic condition at the free boundary, the global existence and uniqueness in one space dimension were established in [21,23] with $g(u)=-u$. The asymptotic behavior of the free boundary was obtained in [6]. Bossavit [2] studied the numerical solution for the model problem associated with classical Stefan conditions on the free boundary. By assuming the magnetic field to be time-harmonic in three space dimensions, the author [29] studied a phase-change problem for a melting process using induction heating. A global weak solution for the phase-change problem is established in [29]. Recently, the author of [28] studied a free boundary problem arising in a microwave heating modelling, where a kinetic condition was used in the model. The general case in $\mathbb{R}^{3}$ was studied in [13] where an enthalpy weak form is used to model the process.

In the present paper we study the phase-change problem (1.1)-(1.10). One of the major difficulties for this problem is that the nonlinear term $\rho h_{x}^{2}$ in (1.2) only belongs to the $L^{1}$-space even without the phase-change. By using a similar idea to that developed in [5, 28] we are able to prove the global solvability for the problem (1.1)-(1.10). One of the key steps in the proof is the $L^{2, \mu}$-theory developed in [30]. Another technique from [1] also plays an important role in the proof of the existence theorem.

This paper is organized as follows. In Section 2, we study the coupled parabolic system when an interface $x=s(t)$ is fixed in a suitable space. The existence of a unique weak solution is established. Moreover, various a priori estimates are derived. These estimates have a precise dependence upon the smoothness of the interface. In Section 3, we use those estimates obtained in Section 2 and Schauder's fixed-point theorem to prove the existence of a solution for the free boundary problem (1.1)-(1.10). The uniqueness is also proved.

\section{A coupled system with discontinuous coefficients}

In this section we study the coupled parabolic system (1.1)-(1.2) subject to appropriate initialboundary conditions and interface conditions when an interface $x=s(t)$ is fixed. We will show that the problem has a unique weak solution. Moreover, we derive various a priori estimates which have a precise dependence upon the smoothness of the interface $x=s(t)$. Those estimates are crucial in order to prove the existence of a solution for the original free boundary problem in Section 3.

Without loss of generality, we assume $L=1$ in (1.5). It is clear that the interface conditions (1.3), (1.5)-(1.6) for $u(x, t)$ are equivalent to the following:

$$
\begin{aligned}
& u(s(t)-, t)=u(s(t)+, t), \quad 0<t<T, \\
& -k_{1} u_{x}(s(t)-, t)+k_{2} u_{x}(s(t)+, t)=-g(u(s(t), t)), \quad 0<t<T, \\
& s^{\prime}(t)=-g(u(s(t), t)), \quad 0<t<T .
\end{aligned}
$$

Throughout this section, we assume that $s \in C^{1}[0, T]$ is fixed. Moreover, $s(0)=s_{0}, 0<s(t)<1$, $0 \leqslant t \leqslant T$. Let $\Gamma_{T}$ be defined as in Section 1 .

Consider the following problem:

$$
\begin{aligned}
& h_{t}-\frac{\partial}{\partial x}\left[\rho(x, t, u) \frac{\partial h}{\partial x}\right]=0, \quad(x, t) \in Q_{T} \backslash \Gamma_{T}, \\
& u_{t}-\frac{\partial}{\partial x}\left[k(x, t, u) \frac{\partial u}{\partial x}\right]=\rho(x, t, u) h_{x}^{2}, \quad(x, t) \in Q_{T} \backslash \Gamma_{T}, \\
& h(s(t)-, t)=h(s(t)+, t), \quad u(s(t)-, t)=u(s(t)+, t), \quad 0<t<T,
\end{aligned}
$$




$$
\begin{aligned}
& \rho_{1} h_{x}(s(t)-, t)=\rho_{2} h_{x}(s(t)+, t), \quad 0<t<T, \\
& -k_{1} u_{x}(s(t)-, t)+k_{2} u_{x}(s(t)+, t)=-g(u(s(t), t)), \quad 0<t<T, \\
& \rho_{1}(0, t, u(0, t)) h_{x}(0, t)=f_{1}(t), \quad 0<t<T, \\
& \rho_{2}(1, t, u(1, t)) h_{x}(1, t)=f_{2}(t), \quad 0<t<T, \\
& u_{x}(0, t)=u_{x}(1, t)=0, \quad 0<t<T, \\
& h(x, 0)=h_{0}(x), \quad u(x, 0)=u_{0}(x), \quad 0<x<1 .
\end{aligned}
$$

Even without free boundary involved in (2.1)-(2.9), it is still a challenge to prove the global existence of a weak solution for (2.1)-(2.9) because of the nonlinear term $\rho(x, t, u) h_{x}^{2}$ in (2.2), the jump condition on the interface in (2.5) and the discontinuous coefficients $k$ and $\rho$ in (2.1)-(2.2). We use the ideas from [5], [24] and [29] to establish the global existence of a unique weak solution.

We introduce the following assumptions:

$\mathrm{H}(2.1): \rho(x, t, u), k(x, t, u)$ are measurable with respect to $(x, t)$ and continuous with respect to $u$ and there exist constants $a_{0}$ and $a_{1}$ such that

$$
0<a_{0} \leqslant \rho(x, t, u), k(x, t, u) \leqslant a_{1} \quad \text { for a.e. }(x, t, u) \in Q_{T} \times \mathbb{R} .
$$

$\mathrm{H}(2.2): g \in C^{1}(\mathbb{R})$ is nonnegative and there exists a constant $G_{0}$ such that

$$
\left|g^{\prime}(s)\right| \leqslant G_{0}, \quad s \in \mathbb{R} .
$$

$\mathrm{H}(2.3): f_{1}, f_{2} \in H^{1}(0, T)$ and $u_{0}, h_{0} \in W^{1, \infty}(0,1)$.

We point out that the assumption on $g$ is stronger than that of [21] due to our method used in this paper. The nonnegativity of $g(s)$ is purely to ensure $s^{\prime}(t) \leqslant 0$, a melting process. It will be seen from the proof of the main result given below that $g$ can be chosen to be of a general form (see Remark 3.1 below).

The main result of this section is the following theorem.

THEOREM 2.1 Under the assumptions $\mathrm{H}(2.1)-(2.3)$ the problem (2.1)-(2.9) has a weak solution $(h, u)$ with

$$
h \in L^{2}\left(0, T ; H^{1}(0,1)\right) \cap C^{\alpha, \alpha / 2}\left(\bar{Q}_{T}\right), \quad u \in L^{2}\left(0, T ; H^{1}(0,1)\right) \cap C^{\alpha, \alpha / 2}\left(\bar{Q}_{T}\right)
$$

for some $\alpha \in(0,1)$. Moreover, the following estimates hold:

$$
\begin{aligned}
& \sup _{0 \leqslant t \leqslant T} \int_{0}^{1}\left[h^{2}+u^{2}\right] \mathrm{d} x+\int_{0}^{T} \int_{0}^{1}\left[h_{x}^{2}+u_{x}^{2}\right] \mathrm{d} x \mathrm{~d} t \leqslant C_{1}, \\
& \|h\|_{C^{\alpha, \alpha / 2}\left(\bar{Q}_{T}\right)}+\|u\|_{C^{\alpha, \alpha / 2}\left(\bar{Q}_{T}\right)} \leqslant C_{2},
\end{aligned}
$$

where $C_{1}$ and $C_{2}$ depend only on the known data, but are independent of the bound of $\|s\|_{C^{1}[0, T]}$.

Proof. We use Schauder's fixed-point theorem to prove the global existence. Let

$$
K=\left\{v \in C^{\beta, \beta / 2}\left(\bar{Q}_{T}\right):\|v\|_{C^{\beta, \beta / 2}\left(\bar{Q}_{T}\right)} \leqslant K_{0}\right\},
$$

where the constants $K_{0}$ and $\beta \in(0,1)$ will be determined later. 
For any $v \in K$, we solve the following parabolic problem:

$$
\begin{aligned}
& h_{t}-\frac{\partial}{\partial x}\left[\rho(x, t, v) \frac{\partial h}{\partial x}\right]=0, \quad(x, t) \in Q_{T}, \\
& \rho_{1}(0, t, v(0, t)) h_{x}(0, t)=f_{1}(t), \quad 0<t<T, \\
& \rho_{2}(1, t, v(1, t)) h_{x}(1, t)=f_{2}(t), \quad 0<t<T, \\
& h(x, 0)=h_{0}(x), \quad 0<x<1 .
\end{aligned}
$$

It is known from [12] that the problem (2.11)-(2.14) has a unique weak solution $h \in$ $L^{2}\left(0, T ; H^{1}(0,1)\right) \cap C^{\alpha_{1}, \alpha_{1} / 2}\left(\bar{Q}_{T}\right)$ for some $\alpha_{1} \in(0,1)$. Note that the weak solution $h$ satisfies the interface conditions (2.3)-(2.4) automatically in the weak sense. Moreover, there exist constants $C_{1}$ and $C_{2}$ such that

$$
\sup _{0 \leqslant t \leqslant T} \int_{0}^{1}|h|^{2} \mathrm{~d} x+\int_{0}^{T} \int_{0}^{1}\left|h_{x}\right|^{2} \mathrm{~d} x \mathrm{~d} t \leqslant C_{1}, \quad\|h\|_{C^{\alpha_{1}, \alpha_{1} / 2}\left(\bar{Q}_{T}\right)} \leqslant C_{2},
$$

where $C_{1}$ and $C_{2}$ depend only on $a_{0}, a_{1}$, the upper bound of $T,\left\|f_{1}\right\|_{H^{1}(0, T)},\left\|f_{2}\right\|_{H^{1}(0, T)}$, and $\left\|h_{0}\right\|_{C^{\alpha}{ }_{10,1]}}$, but are independent of the bound on $K_{0}, \beta$, and the smoothness of $s(t)$.

Next we consider the following parabolic problem:

$$
\begin{aligned}
& u_{t}-\left[k(x, t, u) u_{x}\right]_{x}=\rho(x, t, v(x, t)) h_{x}^{2}, \quad(x, t) \in Q_{T} \backslash \Gamma_{T}, \\
& u_{x}(0, t)=u_{x}(1, t)=0, \quad 0<t<T, \\
& u(s(t)-, t)=u(s(t)+, t), \quad 0<t<T, \\
& k_{1} u_{x}(s(t)-, t)-k_{2} u_{x}(s(t)+, t)=g(u(s(t), t)), \quad 0<t<T, \\
& u(x, 0)=u_{0}(x), \quad 0<x<1 .
\end{aligned}
$$

First of all, we use a technique from [25] employing the Dirac-delta function to absorb the interface conditions into the equation. Since $\rho h_{x}^{2} \in L^{1}\left(Q_{T}\right)$, it is easy to see that the problem (2.15)-(2.19) is equivalent to the following problem in the weak sense, as long as the weak solution is continuous in $\bar{Q}_{T}$ :

$$
\begin{aligned}
& u_{t}-\left[k(x, t, u) u_{x}\right]_{x}+g(u(x, t)) \delta(x-s(t))=\rho(x, t, v(x, t)) h_{x}^{2} \text { in } Q_{T}, \\
& u_{x}(0, t)=u_{x}(1, t)=0, \quad 0<t<T, \\
& u(x, 0)=u_{0}(x), \quad 0<x<1,
\end{aligned}
$$

where $\delta(x-s(t))$ is the Dirac-delta function with mass concentrated at $x=s(t)$.

To obtain a weak solution with desired regularity for (2.20)-(2.22) we have to make a suitable substitution in order to handle the nonlinear term on the right-hand side of (2.20).

We introduce

$$
w(x, t)=u(x, t)+h(x, t)^{2} / 2, \quad(x, t) \in Q_{T} .
$$

By using (2.11) we see that (2.20) is equivalent to the following equation in the weak sense:

$$
w_{t}-\left[k(x, t, u) w_{x}\right]_{x}+g(u) \delta(x-s(t))=\left[(\rho-k) h h_{x}\right]_{x} \quad \text { in } Q_{T} .
$$


Moreover,

$$
\begin{aligned}
& w_{x}(0, t)=\frac{h(0, t) f_{1}(t)}{\rho_{1}(0, t, u(0, t))}, \quad w_{x}(1, t)=\frac{h(1, t) f_{2}(t)}{\rho_{2}(1, t, u(1, t))}, \quad 0<t<T, \\
& w(x, 0)=u_{0}(x)+h_{0}(x)^{2} / 2, \quad 0<x<1,
\end{aligned}
$$

where $u(x, t)=w(x, t)-h(x, t)^{2} / 2$ in $(2.25)$.

Note that for one space dimension the Dirac-delta function can be expressed by the derivative of the Heaviside function in the sense of distributions:

$$
g(u(x, t)) \delta(x-s(t))=g(u(x, t))[H(x-s(t))]_{x} \quad \text { in } Q_{T},
$$

where $H(x)$ is the classical Heaviside function.

Now we can rewrite the singular term in the weak sense:

$$
\begin{aligned}
g(u(x, t)) \frac{\partial H(x-s(t))}{\partial x} & =\frac{\partial}{\partial x}[g(u(x, t)) H(x-s(t))]-H(x-s(t)) g^{\prime}(u(x, t)) u_{x} \\
& =\frac{\partial}{\partial x}[g(u(x, t)) H(x-s(t))]-H(x-s(t)) g^{\prime}(u(x, t))\left[w_{x}-h h_{x}\right] .
\end{aligned}
$$

Since $g^{\prime}(u)$ is uniformly bounded, we see from the theory of parabolic equations ([12]) that the problem (2.24)-(2.26) has a unique weak solution $w \in L^{2}\left(0, T ; H^{1}(0,1)\right)$. Moreover, from the estimate for $h(x, t)$ and $\mathrm{H}(2.1)$ we see that

$$
\left\|\frac{h(0, t) f_{1}(t)}{\rho_{1}}\right\|_{L^{\infty}(0, T)}+\left\|\frac{h(1, t) f_{2}(t)}{\rho_{2}}\right\|_{L^{\infty}(0, T)} \leqslant C,
$$

$I:=H(x-s(t)) g^{\prime}(u) h h_{x} \in L^{2}\left(Q_{T}\right)$ by $\mathrm{H}(2.2)$ and

$$
\iint_{Q_{T}}|I|^{2} \mathrm{~d} x \mathrm{~d} t \leqslant C \iint_{Q_{T}} h_{x}^{2} \mathrm{~d} x \mathrm{~d} t \leqslant C,
$$

where $C$ depends only on the known data, but is independent of $K_{0}$ and the smoothness of $s(t)$. It follows from regularity theory (Theorem 7.1 in [12]) that $w \in C^{\alpha_{2}, \alpha_{2} / 2}\left(\bar{Q}_{T}\right)$ for some $\alpha_{2} \in(0,1)$. Moreover, there exists a constant $C_{3}$ such that

$$
\|w\|_{C^{\alpha_{2}, \alpha_{2} / 2}\left(\bar{Q}_{T}\right)} \leqslant C_{3},
$$

where $C_{3}$ is independent of $\beta, K_{0}$, and the smoothness of $s(t)$.

Define a mapping $M$ from $K$ into $K$ as follows:

$$
M: v \in K \mapsto u:=M[v],
$$

where $u(x, t)=w(x, t)-\frac{1}{2} h(x, t)^{2}$ for $(x, t) \in Q_{T}$ and $w$ is the weak solution of (2.24)-(2.26).

It is clear that the mapping $M$ is well-defined. From the estimates (2.15) and (2.27), we see that

$$
\|u\|_{C^{\alpha, \alpha / 2}\left(\bar{Q}_{T}\right)} \leqslant C_{4},
$$

where $\alpha=\min \left\{\alpha_{1}, \alpha_{2}\right\}$ and $C_{4}$ depends only on $C_{2}, C_{3}$. 
We choose $K_{0}=C_{4}$ and $\beta \in(0, \alpha)$. Then $M$ maps $K$ into $K$. The proof of the continuity of $M$ from $K$ into $K$ is a simpler case of that for the mapping $M_{1}$ defined in Section 3. Hence we skip this step here. Since the embedding from $C^{\alpha, \alpha / 2}\left(\bar{Q}_{T}\right)$ into $C^{\beta, \beta / 2}\left(\bar{Q}_{T}\right)$ is compact for any $0<\beta<\alpha$, it follows that $M$ is compact and continuous from $K$ into $K$. By Schauder's fixed-point theorem, $M$ has a fixed point. This fixed point along with the weak solution $h$ form a weak solution of the problem (2.1)-(2.9).

By using classical regularity theory, we can easily obtain more regularity for the solution of the problem, provided that the known data have more regularity.

Consider the following assumption:

$\mathrm{H}(2.4)$ : (a) $\rho_{i}, k_{i} \in C^{1+\alpha, \alpha, 1+\alpha}\left(Q_{T} \times \mathbb{R}\right)$.

(b) $f_{1}, f_{2} \in C^{1+\alpha}[0, T], u_{0} \in W^{1, \infty}(0,1) \cap C^{2+\alpha}\left[0, s_{0}\right] \cap C^{2+\alpha}\left[s_{0}, 1\right]$.

Moreover, the following consistency condition holds:

$$
k_{1} u_{0}^{\prime}\left(s_{0}-\right)-k_{2} u_{0}^{\prime}\left(s_{0}+\right)=g\left(u_{0}\left(s_{0}\right)\right) .
$$

THEOREM 2.2 Under the assumptions $\mathrm{H}(2.1)-\mathrm{H}(2.4)$ the problem (2.1)-(2.9) a unique classical solution $h, u \in C^{2+\alpha, 1+\alpha / 2}\left(Q_{T}^{ \pm}\right) \cap C^{\alpha, \alpha / 2}\left(\bar{Q}_{T}\right)$. Moreover, $h_{x}$ and $u_{x}$ are continuous up to the interface $x=s(t)$ on $[0, T]$. Furthermore, there exist constants $C_{5}$ and $C_{6}$ such that

$$
\begin{aligned}
& \left\|h_{x}\right\|_{L^{\infty}\left(Q_{T}\right)}+\left\|u_{x}\right\|_{L^{\infty}\left(Q_{T}\right)} \leqslant C_{5}, \\
& \int_{0}^{T}\left\{\int_{0}^{s(t)}\left[u_{x x}^{2}+h_{x x}^{2}\right] \mathrm{d} x+\int_{s(t)}^{1}\left[u_{x x}^{2}+h_{x x}^{2}\right] \mathrm{d} x\right\} \mathrm{d} t \leqslant C_{6},
\end{aligned}
$$

where $C_{5}$ and $C_{6}$ depend on the known data and $\|s\|_{C^{1}[0, T]}$.

Proof. Since $s \in C^{1}[0, T]$, we know from [9] that $u_{x}$ and $h_{x}$ are continuous up to the interface $x=s(t)$. The regularity theory for parabolic equations implies that $h$ and $u$ satisfy the equation in the classical sense in $Q_{T} \backslash \Gamma_{T}$. Moreover, $h_{x}$ and $u_{x}$ are bounded ([9]). The energy method (see [26]) yields the second estimate. Finally, since the solution is classical, the uniqueness follows easily.

\section{The free boundary problem}

We again use Schauder's fixed-point theorem to prove the existence of a solution for the problem (1.1)-(1.10).

Let $\delta>0$ be a small constant and $\beta \in(0, \alpha / 2)$, where $\alpha$ is the Hölder exponent in Theorem 2.2. Let

$$
K_{1}=\left\{s \in C^{1+\beta}[0, T]: s(0)=s_{0}, \delta \leqslant s(t) \leqslant 1-\delta,\|s\|_{C^{1+\beta}[0, T]} \leqslant L_{0}\right\},
$$

where $L_{0}$ is a constant to be determined later. The constant $L_{0}$ depends only on the upper bound of $T$.

For each fixed $s \in K_{1}$, we solve the system (2.1)-(2.9) to obtain a unique solution $(h, u)$ from Theorems 2.1 and 2.2. Define a mapping $M_{1}$ from $K_{1}$ into $C^{1+\alpha / 2}[0, T]$ as follows:

$$
M_{1}: s \in K_{1} \mapsto s^{*}(t)=M_{1}[s](t):=s_{0}-\int_{0}^{t} g(u(s(\tau), \tau)) \mathrm{d} \tau,
$$

where $u$ is the solution of (2.1)-(2.9) corresponding to $s$. 
It is clear from Theorem 2.2 that the mapping $M_{1}$ is well-defined. Moreover, a fixed point of $M_{1}$ along with the solution $(h, u)$ for (2.1)-(2.9) form a solution to the original free boundary problem (1.1)-(1.10).

LEMmA 3.1 Under the assumptions $\mathrm{H}(2.1)-\mathrm{H}(2.4)$ the mapping $M_{1}$ is from $K_{1}$ into $K_{1}$ on $\left[0, T_{0}\right]$ for some $T_{0}>0$, provided that $L_{0}$ is chosen properly.

Proof. From the definition of $s^{*}$ and Hölder continuity of $u$, we have

$$
\left|\left(s^{*}\right)^{\prime}(t)\right|=|g(u(x, t))| \leqslant G_{0}\left|u(x, t)-u_{0}(x)\right|+\left|g\left(u_{0}(x)\right)\right| \leqslant G_{1},
$$

where $G_{1}=2 G_{0} C_{3}+\max _{x \in[0,1]}\left|g\left(u_{0}(x)\right)\right|$. Moreover, we see that for any $t_{1}, t_{2} \in[0, T]$,

$$
\left|\left(s^{*}\right)^{\prime}\left(t_{1}\right)-\left(s^{*}\right)^{\prime}\left(t_{2}\right)\right| \leqslant G_{0}\left|u\left(x, t_{1}\right)-u\left(x, t_{2}\right)\right| \leqslant G_{0} C_{3}\left|t_{1}-t_{2}\right|^{\alpha / 2} .
$$

Furthermore, from the definition of $s^{*}$ there exists a number $T_{0}>0$ such that

$$
0<\delta<s^{*}(t) \leqslant 1-\delta, \quad t \in\left[0, T_{0}\right] .
$$

It follows that $M_{1}$ maps $K_{1}$ into $C^{1+\beta}\left[0, T_{0}\right]$. Moreover, the image of $K_{1}, M_{1}\left(K_{1}\right)$, is a subset of $K_{1}$ if we choose $L_{0}=G_{1}\left[1+C_{3} T_{0}^{\alpha / 2-\beta}\right]+1$.

Since $\beta<\alpha / 2$ and the embedding from $C^{1+\alpha / 2}[0, T]$ into $C^{1+\beta}[0, T]$ is compact, we see that $M_{1}$ is pre-compact if $M_{1}$ is continuous.

To apply Schauder's fixed-point theorem, we only need to show that $M_{1}$ is continuous from $K_{1}$ into $K_{1}$. This is the most complicated step.

LEMmA 3.2 Under the assumptions $\mathrm{H}(2.1)-\mathrm{H}(2.4)$ the mapping $M_{1}$ is continuous from $K_{1}$ into $K_{1}$.

Proof. Fix any sequence $s_{n} \in K_{1}$ with $s_{n} \rightarrow s$ in $C^{1+\beta}[0, T]$. We denote by $\left(h_{n}, u_{n}\right)$ and $(h, u)$, respectively, the solutions of (2.1)-(2.9) from Theorem 2.1, corresponding to $x=s_{n}$ and $x=s$. Let $s_{n}^{*}=M_{1}\left[s_{n}\right]$ and $s^{*}=M_{1}[s]$.

From Theorem 2.1, we know that the estimates (2.10)-(2.11) in Theorem 2.1 hold uniformly for $\left(h_{n}, u_{n}\right)$. Moreover, since

$$
\left\|s_{n}\right\|_{C^{1+\beta}[0, T]} \leqslant K_{0}, \quad\|s\|_{C^{1+\beta}[0, T]} \leqslant K_{0},
$$

it follows that the estimates (2.28)-(2.29) in Theorem 2.2 also hold for $\left(h_{n}, u_{n}\right)$ and $(h, u)$.

Now we use a transformation similar to the one employed in [1]. Let

$$
\psi:[0,1] \times[\delta, 1-\delta] \rightarrow[-1,1]
$$

be a function such that

(i) $\psi \in C^{3}([0,1] \times[\delta, 1-\delta])$ and $\left|D^{\gamma} \psi\right| \leqslant d_{0}$ for all multi-indices $\gamma=\left(\gamma_{1}, \gamma_{2}\right)$ with $|\gamma|:=$ $\left|\gamma_{1}\right|+\left|\gamma_{2}\right| \leqslant 3$

(ii) $\psi(0, s)=0, \psi(1, s)=1, \psi(s, s)=0$, for all $s \in[\delta, 1-\delta]$;

(iii) $\psi_{x}(s, s)=1 / 2, \psi_{x}(x, s) \geqslant d_{1}>0$, for all $(x, s) \in[0,1] \times[\delta, 1-\delta]$,

where $d_{0}$ and $d_{1}$ are constants. 
Let $\xi=\psi(x, s(t)), 0 \leqslant t \leqslant T$ and $Q_{T}^{*}=(-1,1) \times(0, T]$. We use $(\xi, t)$ as new variables and set $I(\xi, t)=h(x, t), v(\xi, t)=u(x, t)$. It is clear that

$$
h_{t}=I_{t}+I_{\xi} \psi_{s} s^{\prime}, \quad h_{x}=I_{\xi} \psi_{x}, \quad h_{x x}=I_{\xi \xi}\left(\psi_{x}\right)^{2}+I_{\xi} \psi_{x x} .
$$

Similarly, one can calculate $v_{t}, v_{\xi}$ and $v_{\xi \xi}$.

Let

$$
S^{-}=(-1,0) \times(0, T], \quad S^{+}=(0,1) \times(0, T] .
$$

A direct calculation shows that $I(\xi, t)$ and $v(\xi, t)$ satisfy the following equations:

$$
\begin{array}{ll}
I_{t}-\frac{\partial}{\partial \xi}\left[\rho_{1}(x, t, u) \psi_{x} I_{\xi}\right] \psi_{x}=J_{1}(\xi, t, I, v, s), & (\xi, t) \in S^{-}, \\
I_{t}-\frac{\partial}{\partial \xi}\left[\rho_{2}(x, t, u) \psi_{x} I_{\xi}\right] \psi_{x}=J_{2}(\xi, t, I, v, s), & (\xi, t) \in S^{+}, \\
v_{t}-\frac{\partial}{\partial \xi}\left[k_{1}(x, t, u) \psi_{x} v_{\xi}\right] \psi_{x}=J_{3}(\xi, t, I, v, s), & (\xi, t) \in S^{-}, \\
v_{t}-\frac{\partial}{\partial \xi}\left[k_{2}(x, t, u) \psi_{x} v_{\xi}\right] \psi_{x}=J_{4}(\xi, t, I, v, s), & (\xi, t) \in S^{+},
\end{array}
$$

where

$$
\begin{array}{ll}
J_{1}(\xi, t, I, v, s):=-\psi_{s} s^{\prime} I_{\xi}, & J_{2}(\xi, t, I, v, s):=-\psi_{s} s^{\prime} I_{\xi}, \\
J_{3}(\xi, t, I, v, s):=\rho_{1}\left[I_{\xi} \psi_{x}\right]^{2}-\psi_{s} s^{\prime} v_{\xi}, & J_{4}(\xi, t, I, v, s):=\rho_{2}\left[I_{\xi} \psi_{x}\right]^{2}-\psi_{s} s^{\prime} v_{\xi} .
\end{array}
$$

On the interface $\xi=0,0 \leqslant t \leqslant T$,

$$
\begin{aligned}
& h(0-, t)=h(0+, t) \\
& \rho_{1}(0-, t, v(0, t)) h_{\xi}(0-, t)=\rho_{2}(0+, t, v(0, t)) h_{\xi}(0+, t) \\
& v(0-, t)=v(0+, t) \\
& k_{1}(0, t, v(0, t)) v_{\xi}(0-, t)-k_{2}(0, t, v(0, t)) v_{\xi}(0+, t)=\frac{g(v(0, t))}{\psi_{x}} .
\end{aligned}
$$

We also introduce the same transformation $\xi=\psi\left(x, s_{n}(t)\right)$ corresponding to the solution $\left(h_{n}, u_{n}, s_{n}\right)$ and define

$$
I_{n}(\xi, t)=h_{n}(x, t), \quad v_{n}(\xi, t)=u_{n}(x, t), \quad(x, t) \in Q_{T} .
$$

Then $I_{n}(\xi, t)$ and $v_{n}(\xi, t)$ satisfy the same set of equations and the boundary conditions as do $I(\xi, t)$ and $v(\xi, t)$ in $S^{ \pm}$.

Set

$$
P(\xi, t)=I(\xi, t)-I_{n}(\xi, t), \quad U(\xi, t)=v(\xi, t)-v_{n}(\xi, t), \quad(\xi, t) \in S^{ \pm}
$$

Then a direct calculation shows that $P(\xi, t)$ satisfies the following equation in the weak sense:

$$
P_{t}-\frac{\partial}{\partial \xi}\left[\rho_{1}(x, t, u) \psi_{x} P_{\xi}\right] \psi_{x}=J_{1}-J_{1 n}+R_{1} \quad \text { in } S^{-},
$$




$$
P_{t}-\frac{\partial}{\partial \xi}\left[\rho_{2}(x, t, u) \psi_{x} P_{\xi}\right] \psi_{x}=J_{2}-J_{2 n}+R_{2} \quad \text { in } S^{+},
$$

where

$$
\begin{aligned}
& R_{1}=\frac{\partial}{\partial \xi}\left\{\left[\rho_{1}(x, t, u) \psi_{x}-\rho_{1}\left(x, t, u_{n}\right) \psi_{n x}\right] I_{n \xi}\right\} \psi_{x}+\frac{\partial}{\partial \xi}\left[\rho_{1} \psi_{n x} I_{n \xi}\right]\left(\psi_{x}-\psi_{n x}\right), \\
& R_{2}=\frac{\partial}{\partial \xi}\left\{\left[\rho_{2}(x, t, u) \psi_{x}-\rho_{2}\left(x, t, u_{n}\right) \psi_{n x}\right] I_{n \xi}\right\} \psi_{x}+\frac{\partial}{\partial \xi}\left[\rho_{2} \psi_{n x} I_{n \xi}\right]\left(\psi_{x}-\psi_{n x}\right) .
\end{aligned}
$$

Similarly, we can derive the equations for $U(\xi, t)$ in $S^{ \pm}$.

Since $\psi_{x}, \psi_{s}, \psi_{n x}, \psi_{n s}, I_{\xi}, I_{n \xi}, s^{\prime}, s_{n}^{\prime}$ are uniformly bounded, from the equations for $P(x, t)$ and $U(x, t)$ and the estimates (2.28)-(2.29) we can derive the following energy estimates:

$$
\begin{aligned}
\int_{-1}^{1}|P|^{2} \mathrm{~d} \xi+\int_{0}^{T} \int_{-1}^{1}\left|P_{\xi}\right|^{2} \mathrm{~d} \xi \mathrm{d} t & \leqslant \delta \int_{0}^{T} \int_{-1}^{1}\left[\left|P_{\xi}\right|^{2}+\left|U_{\xi}\right|^{2}\right] \mathrm{d} \xi \mathrm{d} t \\
& +C(\delta) \int_{0}^{T} \int_{-1}^{1}\left[P^{2}+U^{2}\right] \mathrm{d} \xi \mathrm{d} t+C(\delta) \int_{0}^{T}\left|s_{n}^{\prime}(t)-s^{\prime}(t)\right|^{2} \mathrm{~d} t \\
\int_{-1}^{1}|U|^{2} \mathrm{~d} \xi+\int_{0}^{T} \int_{-1}^{1}\left|U_{\xi}\right|^{2} \mathrm{~d} \xi \mathrm{d} t & \leqslant \delta \int_{0}^{T} \int_{-1}^{1}\left[\left|P_{\xi}\right|^{2}+\left|U_{\xi}\right|^{2}\right] \mathrm{d} \xi \mathrm{d} t \\
& +C(\delta) \int_{0}^{T} \int_{-1}^{1}\left[P^{2}+U^{2}\right] \mathrm{d} \xi \mathrm{d} t+C(\delta) \int_{0}^{T}\left|s_{n}^{\prime}(t)-s^{\prime}(t)\right|^{2} \mathrm{~d} t
\end{aligned}
$$

where $\delta>0$ is a small parameter.

Since $T$ in the above estimates can be replaced by any $T^{*} \in(0, T]$, by first choosing $\delta$ sufficiently small and then using Gronwall's inequality we obtain

$$
\int_{-1}^{1}\left[P^{2}+U^{2}\right] \mathrm{d} \xi+\int_{0}^{T} \int_{-1}^{1}\left[\left|P_{\xi}\right|^{2}+\left|U_{\xi}\right|^{2}\right] \mathrm{d} \xi \mathrm{d} t \leqslant C \int_{0}^{T}\left|s^{\prime}(t)-s_{n}^{\prime}(t)\right|^{2} \mathrm{~d} t .
$$

It follows that $h_{n} \rightarrow h$ and $u_{n} \rightarrow u$ in $L^{2}\left(0, T ; H^{1}(\Omega)\right)$ as $n \rightarrow \infty$.

Now we use $L^{2, \mu}$-theory (see [20]) to show that $u_{n}$ converges to $u$ in $C^{\alpha, \alpha / 2}\left(\bar{Q}_{T}\right)$ as $n \rightarrow \infty$. First of all, from $L^{2, \mu}$-theory for any $\mu \in(0,1)$ (see [30]) we have

$$
\begin{aligned}
\left\|P_{x}\right\|_{L^{2, \mu}\left(Q_{T}^{*}\right)} & \leqslant C\left\|s_{n}^{\prime}-s^{\prime}\right\|_{L^{\infty}(0, T)}+\|P\|_{L^{2}\left(0, T ; H^{1}(-1,1)\right)}+\|U\|_{L^{2}\left(0, T ; H^{1}(-1,1)\right)} \\
& \leqslant C\left\|s-s_{n}\right\|_{W^{1, \infty}(0, T)} .
\end{aligned}
$$

Next we use the same technique as in Section 2 to rewrite the nonlinear term $\rho_{i} P_{\xi}^{2}, i=1,2$, in divergence form and then introduce $W(\xi, t)=U(\xi, t)+P(\xi, t)^{2} / 2$. For the equation for $W(\xi, t)$, we apply $L^{2, \mu}$-theory to obtain

$$
\begin{aligned}
\left\|W_{x}\right\|_{L^{2, \mu}\left(Q_{T}^{*}\right)} & \leqslant C\left[\left\|P_{x}\right\|_{L^{2, \mu}\left(Q_{T}^{*}\right)}+\|P\|_{L^{2}\left(0, T ; H^{1}(-1,1)\right)}+\|U\|_{L^{2}\left(0, T ; H^{1}(-1,1)\right)}\right] \\
& \leqslant C\left\|s-s_{n}\right\|_{W^{1, \infty}(0, T)} .
\end{aligned}
$$

Finally, by applying parabolic interpolation we obtain

$$
\|P\|_{C^{\alpha, \alpha / 2}\left(Q_{T}^{*}\right)}+\|W\|_{C^{\alpha, \alpha / 2}\left(Q_{T}^{*}\right)} \leqslant C\left\|s-s_{n}\right\|_{W^{1, \infty}(0, T)},
$$

which approaches 0 as $n \rightarrow \infty$ since $s_{n} \rightarrow s$ in $C^{1+\beta}[0, T]$. 
Thus, from the definition of $W$ we see that $u_{n}$ converges to $u$ in $C^{\alpha, \alpha / 2}\left(\bar{Q}_{T}\right)$, which implies that the mapping $M_{1}$ is continuous from $K_{1}$ into $K_{1}$.

Now we use Lemmas 3.1-3.2 and Schauder's fixed-point theorem to obtain the following main result.

ThEOREM 3.3 Under the assumptions $\mathrm{H}(2.1)-\mathrm{H}(2.4)$ the problem (1.1)-(1.10) has a unique classical solution in $Q_{T}$ for some $T>0$.

Proof. The existence follows from Schauder's fixed-point theorem. Indeed, from Lemmas 3.1 and 3.2 the mapping $M_{1}$ is from $K_{1}$ into $K_{1}$. Moreover, since the image of $K_{1}$ under $M_{1}$ is a subset of $C^{1+\alpha / 2}[0, T]$ and the embedding from $C^{1+\alpha / 2}[0, T]$ into $C^{1+\beta}[0, T]$ is compact when $0<\beta<$ $\alpha / 2$, it follows that $M_{1}$ is also compact. Schauder's fixed-point theorem implies that $M_{1}$ has a fixed point $s=M[s]$. This fixed point $s$ along with $(h, u)$ form a solution of the original problem (1.1)-(1.10).

To show the uniqueness, we use the same argument as in Lemma 3.2. Suppose $\left(h_{1}, u_{1}, s_{1}\right)$ and $\left(h_{2}, u_{2}, s_{2}\right)$ are two solutions of the problem (1.1)-(1.10). Let

$$
h(x, t)=h_{1}(x, t)-h_{2}(x, t), \quad u(x, t)=u_{1}(x, t)-u_{2}(x, t), \quad s(t)=s_{1}(t)-s_{2}(t) .
$$

By introducing the same new variables $\xi=\psi(x, s)$ as in Lemma 3.2, we can easily derive the estimate

$$
\|P\|_{L^{2}\left(0, T ; H^{1}(-1,1)\right)}+\|U\|_{L^{2}\left(0, T ; H^{1}(-1,1)\right)} \leqslant C \int_{0}^{T}\left|s_{1}^{\prime}(t)-s_{2}^{\prime}(t)\right|^{2} \mathrm{~d} t,
$$

where $P(\xi, t)=h(x, t)$ and $U(\xi, t)=u(x, t)$. Using the free boundary condition (1.6) and the Sobolev interpolation, we have

$$
\begin{aligned}
\left|s_{1}^{\prime}(t)-s_{2}^{\prime}(t)\right| & =\left|g\left(u_{1}\left(s_{1}(t), t\right)\right)-g\left(u_{2}\left(s_{2}(t), t\right)\right)\right| \leqslant C|U(0, t)| \\
& \leqslant \varepsilon \int_{-1}^{1}\left|U_{\xi}\right|^{2} \mathrm{~d} \xi+C(\varepsilon) \int_{-1}^{1} U^{2} \mathrm{~d} \xi,
\end{aligned}
$$

where $\varepsilon$ is a small parameter. After choosing $\varepsilon$ properly and using Gronwall's inequality, we obtain

$$
\int_{-1}^{1}\left[|P|^{2}+|U|^{2}\right] \mathrm{d} \xi=0,
$$

which yields the uniqueness.

Now we show that the solution $(h(x, t), u(x, t), s(t))$ can be extended to any interval $[0, T]$ for any $T>0$.

ThEOREM 3.4 Under the conditions $\mathrm{H}(2.1)-(2.4)$, the solution $(h, u, s)$ can be extended to any interval $[0, T]$, where $s(t)=0$ if the interface $x=s(t)$ intersects the fixed boundary $x=0$.

Proof. Suppose $T>0$ is arbitrary. From the above proof, we see that the solution $(h, u, s)$ can be extended as long as the free boundary $x=s(t)$ stays away from the fixed boundary $x=0$. If $s(t)$ stays away from the fixed boundary $x=0$ on $[0, T]$, then the conclusion holds from Theorem 3.3. On the other hand, suppose $x=s(t)$ intersects the fixed boundary $x=0$, say, at $t=t^{*}<T$. Namely,

$$
\lim _{t \rightarrow t^{*}-} s(t)=0,
$$

where the existence of the limit is guaranteed by the uniform boundedness of $s(t)$ in $C^{1+\beta}\left[0, t^{*}\right)$. 
Note that

$$
s^{\prime}(t)=-g(u(s(t), t)), \quad 0<t<t^{*},
$$

is uniformly bounded on $\left[0, t^{*}\right)$. Moreover, $h$ and $u$ are Hölder continuous in $Q_{t^{*}}$. It follows that

$$
\lim _{t \rightarrow t^{*}} h(\cdot, t)=h\left(\cdot, t^{*}\right) \in C^{\alpha}[0,1]
$$

and

$$
\lim _{t \rightarrow t^{*}} u(\cdot, t)=u\left(\cdot, t^{*}\right) \in C^{\alpha}[0,1] .
$$

Moreover, from the regularity theory for parabolic equations, we know that $h\left(\cdot, t^{*}\right), u\left(\cdot, t^{*}\right) \in$ $C^{\alpha}[0,1] \cap C^{2+\alpha}(0,1)$. Now we can use $h\left(x, t^{*}\right)$ and $u\left(x, t^{*}\right)$ as new initial values and consider the problem (1.1)-(1.2) associated with the same initial-boundary conditions in $\left[t^{*}, T\right]$ (no interface conditions are involved). By using the same method as in Section 2, we see that this problem has a unique solution on $\left[t^{*}, T\right]$.

If the coefficients in (1.1)-(1.2) are smooth, then we can obtain the smoothness of the solution.

ThEOREM 3.5 Let $0<s(t)<1$ on [0,T]. Suppose all coefficients in (1.1)-(1.2) are smooth and $g \in C^{\infty}(\mathbb{R})$. Then $h$ and $u$ are smooth in $Q_{T}^{ \pm}$and $s \in C^{\infty}(0, T)$.

Proof. The idea of the proof comes from [9, 19]. From the interface condition (1.6), we know that $s \in C^{2+\alpha / 2}(0, T]$. From the regularity theory for parabolic equations we see that $h$ and $u$ are in $C^{2,1+\alpha / 2}\left(Q_{T}^{ \pm}\right)$. Moreover, $u_{x x}$ is continuous up to the interface $x=s$. This implies that $s \in C^{3+\alpha / 2}(0, T]$. Then we use the regularity theory again to find that $u_{x x x}$ is continuous up to $x=s(t)$. By continuing this process, we see that $s \in C^{\infty}(0, T]$.

REMARK 3.1 It is easy to see that the results in this paper hold for a more general free-boundary condition with suitable assumptions:

$$
s^{\prime}(t)=-g(s(t), u(s(t), t)), \quad 0 \leqslant t \leqslant T .
$$

\section{Acknowledgments}

The author would like to thank Professor R. Dillon and Professor D. Wollkind for their careful reading of the paper and useful comments. Many thanks also go to the anonymous referees for their suggestions which improved the original version of the paper.

\section{REFERENCES}

1. Baiocchi, C., Evans, L. C., Frank, L., \& Friedman, A. Uniqueness for two immiscible fluids in a one-dimensional porous medium. J. Differential Equations 36 (1980), 249-256. Zbl 0454.76081 MR 0574338

2. Boss avit, A. Mixed methods for a vectorial Stefan problem. Free Boundary Problems: Theory and Applications, Vol. I (Irsee, 1987), Pitman Res. Notes Math. Ser. 185, Longman Sci. Tech., Harlow (1990), 25-37. MR 1077030 Zbl 0724.65118

3. Bossavit, A., \& Rodrigues, J.-F. On the electromagnetic "induction heating" problem in bounded domains. Adv. Math. Sci. Appl. 4 (1994), 79-92. Zbl 0813.35120 MR 1287908 
4. Caginalp, G. An analysis of a phase field model of a free boundary. Arch. Ration. Mech. Anal. 92 (1986), 205-245. Zbl 0608.35080 MR 0816623

5. Chadam, J. M., \& Yin, H. M. A diffusion equation with localized reactions. Proc. Edinburgh Math. Soc. 37 (1994), 101-118. Zbl 0790.35045 MR 1258034

6. Dewynne, J. N., Howison, S. D., Ockendon, J. R., \& Xie, W. Asymptotic behavior of solutions to the Stefan problem with a kinetic condition at the free bounfary. J. Austral. Math. Soc. Ser. B 31 (1989), 81-96. Zbl 0713.35102 MR 1002093

7. DiBenedetto, E., \& Friedman, A. The ill-posed Hele-Shaw model and the Stefan problem for supercooled water. Trans. Amer. Math. Soc. 282 (1984), 183-201. Zbl 0621.35102 MR 0728709

8. Fasano, A., Primicerio, M., \& LaCey, A. A. New results on some classical parabolic free boundary problems. Quart. Appl. Math. 38 (1981), 439-460. Zbl 0468.35081 MR 0614552

9. JIANG, L. S. Existence and differentiability of the solution of a two-phase Stefan problem for quasilinear parabolic equations. Acta Math. Sinica 15 (1965), 749-764. Zbl 0149.31503

10. KANG, K., \& KIM, S. On the Hölder continuity of solutions of a certain system related to Maxwell's equations. SIAM J. Math. Anal. 34 (2002), 87-100. Zbl 1055.35125 MR 2139568

11. Landau, L. D., \& Lifshitz, E. M. Electrodynamics of Continuous Media. Pergamon Press, New York (1960). Zbl 0122.45002 MR 0121049

12. Ladyženskaja, O. A., Solonnikov, V. A., \& Ural'ceva, N. N. Linear and Quasi-Linear Equations of Parabolic Type. Amer. Math. Soc. Transl. 23, Amer. Math. Soc., Providence, RI (1968). Zbl 0174.15403 MR 0241822

13. Manoranjan, R. V., Yin, H. M., \& Showalter, R. On two-phase Stefan problem arising from a microwave heating process. Discrete Contin. Dynam. Systems 15 (2006), 1155-1168. Zbl 1129.35085 MR 2224502

14. Meirmanov, A. M. The Stefan Problem. de Gruyter, New York (1992). Zbl 0751.35052 MR 1154310

15. Metaxas, A. C. Foundations of Electroheat. A Unified Approach. Wiley, New York (1996).

16. Metaxas, A. C., \& Meredith, R. J. Industrial Microwave Heating. I.E.E. Power Engrg. Ser. 4, Per Peregrimus Ltd., London (1983).

17. Miranville, A., Showalter, R., \& Yin, H. M. (eds.), Discrete Contin. Dynam. Systems, a special issue on free boundary problems, 15 (2006), no. 4.

18. Rubinstein, L. I. The Stefan Problem. Amer. Math. Soc., Providence, RI (1971). MR 0351348

19. SCHAEFFER, D. G. A new proof of the infinite differentiablity of the free boundary in the Stefan problem. J. Differential Equations 20 (1976), 266-269. Zbl 0314.35044 MR 0390499

20. Troianiello, G. M. Elliptic Differential Equations and Obstacle Problems. Plenum Press, New York (1987). MR 1094820 Zbl 0655.35002

21. Visintin, A. Stefan problem with a kinetic condition at the free boundary. Ann. Mat. Pura Appl. 146 (1987), 97-122. Zbl 0643.35115 MR 0916689

22. Visintin, A. Supercooling and superheating effects in phase transitions. IMA J. Appl. Math. 35 (1985), 233-256. MR 0839201 Zbl 0615.35090

23. XIE, W. The Stefan problem with a kinetic condition at the free boundary. SIAM J. Math. Anal. 21 (1990), 362-373. Zbl 0737.35165 MR 1038897

24. YIN, H. M. Optimal regularity of solution to a degenerate elliptic system arising in electromagnetic fields. Comm. Pure Appl. Anal. 1 (2002), 127-134. Zbl 1010.35038 MR 1877670

25. YIN, H. M. Blowup and global existence for a non-equilibrium phase change process. Variational and Free Boundary Problems, A. Friedman and J. Spruck (eds.), Springer, New York (1993), 195-204. Zbl 0798.35169 MR 1320782 
26. YIN, H. M. On a free boundary problem with superheating arising in microwave heating processes. Adv. Math. Sci. Appl. 12 (2002), 409-433. Zbl 1033.35157| MR 1909455

27. YIN, H. M. On a phase-field model for a melting problem arising in induction heating processes. Nonlinear Partial Differential Equations and Their Applications, GAKUTO Int. Ser. Math. Sci. Appl. 20, Gakkōtosho, Tokyo (2004), 368-375. Zbl pre02131312 MR 2087483

28. YIN, H. M. On a nonlinear Maxwell's system in quasi-stationary electromagnetic fields. Math. Methods Appl. Sci. 14 (2004), 1521-1539. Zbl 1072.35183 MR 2095301

29. YIN, H. M. On a phase-change problem arising from inductive heating. Nonlinear Differential Equations Appl. 13 (2007), 735-757. Zbl 1128.35107 MR 2329027

30. YIN, H. M. $L^{2, \mu}$-theory for parabolic equations. J. Partial Differential Equations 10 (1997), 31-44. Zbl 0891.35021 MR 1443569 